\title{
Détermination et niveau des activités lipolytiques dans les fromages à pâte persillée
}

\author{
par \\ G. LAMBERET* et A. MENASSA*
}

\section{Rés u m é}

L'activité lipolytique de suspensions de fromages de type Bleu (F) a été mesurée à $\mathrm{pH} 8,0$ sur émulsion de tributyrine et à $\mathrm{pH} 5,5$ sur émulsion de tricaproïne ; l'activité du mycélium de Penicillium roqueforti prélevé dans la pâte (M) a été mesurée sur les deux substrats aux pH 4,5-5,5 et 8,0. Sept échantillons dont cinq Roquefort ont été ainsi analysés.

L'activité à $\mathrm{pH} 5,5$ est la seule mesurable pour (F) dans les Roquefort ; elle est plus forte que celle à $\mathrm{pH} 8,0$ pour un Bleu Bavarois et un Saingorlon. Les valeurs obtenues pour (M) sont également, à une exception près, plus fortes sur tricaproïne à $\mathrm{pH} 5,5$. A partir des diagrammes présentant les activités sur chacun des substrats aux différents $\mathrm{pH}$, il est conclu que les valeurs obtenues résultent de la somme des activités respectives des lipases acide et alcaline de $P$. roquefort $i$.

En utilisant, comme coefficients, les rapports des activités en fonction $\mathrm{du} \mathrm{pH}$ et de la nature du substrat déterminés antérieurement pour les deux enzymes, un système de deux équations a été établi pour calculer les quantités de l'une et l'autre lipases dans le mycélium, sur la base des résultats à $\mathrm{pH} 5,5$ sur tricaproïne et à pH 8,0 sur tributyrine. Ainsi, il apparaît que la lipase alcaline est absente dans trois échantillons et est présente en quantité plus importante que la lipase acide dans le mycélium d'un seul d'entre-eux.

Mots clés :

Lipases - Détermination - Fromages - Penicillium roqueforti.

* Laboratoire de Recherches de la Chaire de Technologie (I.N.R.A.), Institut National Agronomique Paris-Grignon - 78850 Thiverval-Grignon. 


\section{S u m m a ry}

Lipolytic activities of seven samples of Blue veined cheeses, among them five Roquefort cheeses, were determined with synthetic triglycerides as substrate. Enzymatic extracts were cheese suspensions $(C)$ and suspensions of Pencillium roqueforti mycelium (M) gathered on the same cheeses. Measurements were carried out at $20^{\circ} \mathrm{C}$ at constant $\mathrm{pH}$ ( $\mathrm{pH}$-stat method) on emulsions of tributyrin at $p H 8.0$ and tricaproin at $p H 5.5$ for $(C)$ and on both substrates at $p H 4.5,5.5$ and 8.0 for $(M)$.

At $p H 5.5$, results for $(C)$ range between 0.1 and 0.34 L.U. (1 lipase unit $=1$ ueq. of acid released $/ \mathrm{min})$ in Roquefort samples; the values reach 0.61 and 1.27 for Bleu Bavarois and Saingorlon cheeses respectively. At $\mathrm{pH} 8.0$, only these two cheeses present a measurable activity of 0.4 and 0.3 U.L./g respectively.

Values obtained for (M) confirm that all activities excepted one are highest with tricaproin at $p H$ 5.5; at $p H 4.5$ and 8.0 they are between 40 and $70 \%$. Diagrams showing activities on both substrates at different $\mathrm{pH}$ lead to suppose that the total activity could be considered as the resultant of activities of $\mathrm{P}$. roqueforti acid and alkaline lipases previously characterized. Using as coefficients the $p H$ and substrate activity ratios which have been obtained with both enzymes, a system with two equations had been established for estimating their respective amounts on the basis of results at $p H 5.5$ on tricaproin and $p H 8.0$ on tributyrin. It appears then that the alkaline lipase seems missing in 3 samples; it is found in more important amount than acid lipase only in the mycellium of one of them. The observed differences could be the result of lipolytic abilities characterizing P. roqueforti strains and also probably the result of evolution during cheese ripening of the "medium" conditions, particularly the $\mathrm{pH}$, which are liable to influence the mould metabolism.

A better knowledge of the action of both enzymes in the curd will be necessary to point out their influences on organoleptic quality development of cheeses.

Key words:

Lipases - Determination - Cheese - Penicillium roqueforti.

Les fromages à pâte persillée, affinés par la moisissure Penicillium roqueforti, sont ceux dans lesquels la lipolyse est, en général, la plus forte (Vanbelle et al., 1978). Diverses études ont cependant montré qu'il existait des différences importantes quant aux niveaux de la lipolyse et aux profils d'acides gras libres ; ces différences peuvent résulter en partie de la nature du lait (vache ou brebis) mais aussi du choix des souches de la moisissure ensemencée (Anderson et Day, 
1965 ; Fujishima et al., 1971 ; Hall et Kosikowski, 1970 ; Niki et al., 1966).

On sait que $P$. roqueforti produit deux lipases exo- ou endocellulaires qui se distinguent par leurs $\mathrm{pH}$ optimaux d'action, acide ou alcalin (Morris et Jezeski, 1953). L'étude des caractères de ces enzymes montre une différence de spécificité sur les triglycérides simples, la lipase acide est plus active sur la tricaproïne, la lipase alcaline sur la tributyrine (Menassa et Lamberet, 1982), cette différence de spécificité s'exprimant également sur la matière grasse de lait (Imamura et Kataoka, 1963).

Il est donc apparu nécessaire, suite aux essais de purification et de caractérisation des enzymes produites en milieu liquide (Menassa et Lamberet, 1982; Lamberet et Menassa, 1983) de déterminer la nature des activités lipolytiques présentes dans les fromages de type Bleu et, si possible, d'en apprécier les niveaux relatifs afin de mieux comprendre le phénomène de lipolyse dans ce type de pâte.

\section{PROTOCOLE EXPERIMENTAL}

\section{Matériel d'étude}

L'analyse a porté sur sept fromages de type Bleu : trois échantillons (Bleu Bavarois, Saingorlon et Roquefort), d'un poids d'environ $300 \mathrm{~g}$, ont été achetés dans le commerce. Les quatre autres nous ont été fournis par la Société des Caves de Roquefort : ils correspondent à des fromages ensemencés avec deux souches pures différentes de Penicillium roqueforti, souches 1 et 2 , et affinés pendant 20 ou 47 semaines ; les échantillons ayant été prélevés au même moment, les fromages diffèrent par leurs époques de fabrication. Pour ces Roquefort, l'échantillon analysé correspond au mélange de deux tranches prélevées sur un demi fromage.

\section{Préparation des suspensions de fromages}

Sur une première partie de chaque échantillon, la zone présentant des cavités colonisées par la moisissure est séparée de la périphérie (marge de 1 à $1,5 \mathrm{~cm}$ d'épaisseur). L'activité n'a été déterminée que dans la zone colonisée, des essais ayant montré que les valeurs obtenues pour la zone périphérique n'étaient pas différentes des blancs. La partie interne est mise en suspension à $20 \%$ (en poids) en tampon citrate $0,01 \mathrm{M}$ à $\mathrm{pH} 6,0$ par agitation à l'Ultra-Turrax, pendant $3 \mathrm{~min}$ à la vitesse maximale de l'appareil, à température inférieure à $10^{\circ} \mathrm{C}$. Les activités lipolytiques sont déterminées sur cette suspension ; les résultats sont exprimés en unités lipase ( 1 U.L. $=1 \mu$ éq. acide libéré par mn) par gramme de pâte, pour l'ensemble du fromage, en tenant compte des proportions pondérales des deux zones séparées. 
TABLEAU 1 - TABLE 1

Activité lipolytique de fromages à pâte persillée - Lipolytic activities in blue veined cheeses

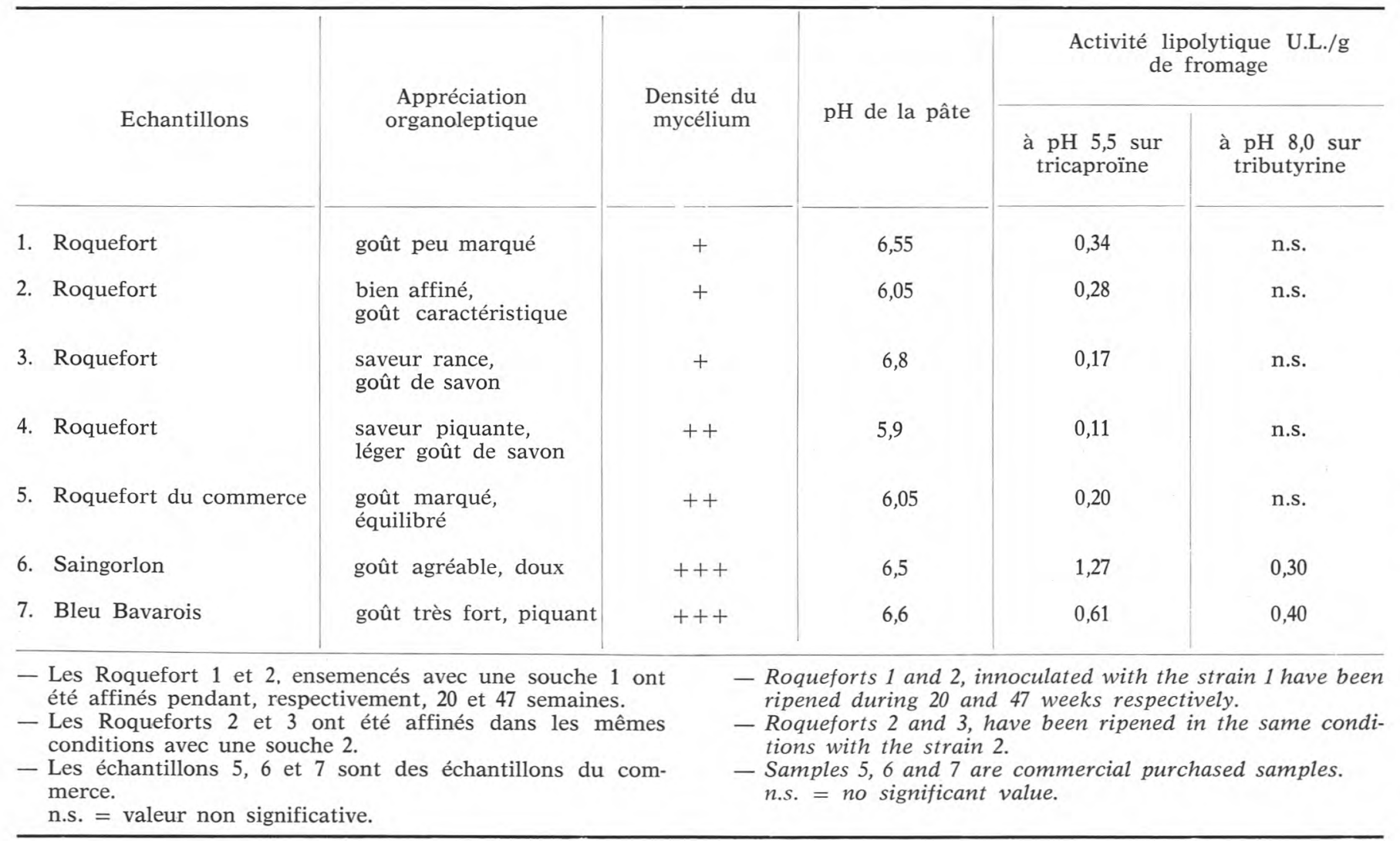




\section{Préparation des suspensions de mycélium}

Sur une seconde partie des échantillons, une quantité de mycélium, variant de 0,5 à $1 \mathrm{~g}$, est prélevée avec une spatule métallique dans les cavités en prenant soin d'entraîner un minimum de pâte. Une suspension à $20 \%$ est réalisée dans les conditions identiques à celles décrites pour le fromage et l'activité est exprimée en U.L./g de mycélium.

\section{Mesure des activités lipolytiques}

Les activités lipolytiques sont mesurées au pH-stat sur tributyrine ou sur tricaproïne selon les conditions précisées antérieurement (Menassa et Lamberet, 1982). La prise d'essai est de $1 \mathrm{ml}$ pour la suspension de fromage, 0,1 ou $0,2 \mathrm{ml}$ pour celle de mycélium. Les mesures sont réalisées en double à $20^{\circ} \mathrm{C}$ aux pH 4,5-5,5 et 8,0 ; les résultats sont exprimés en unités lipase, après correction éventuelle des essais à blanc (extraits enzymatiques dénaturés par la chaleur) et du pourcentage d'acide gras titré.

\section{RESULTATS}

\section{Fromage}

Les résultats de l'activité lipolytique sont groupés dans le tableau 1. A $\mathrm{pH} 8,0$, sur tributyrine, les résultats sont trop faibles pour être significativement différents des essais à blanc sauf pour les échantillons 6 et 7 où le mycélium était abondant et régulièrement réparti. les valeurs sont alors de 0,30 et 0,40 U.L./g de fromage humide. L'activité sur tricaproïne à $\mathrm{pH} 5,5$ est appréciable mais très variable d'un échantillon à l'autre : de 0,11 à 1,27 U.L./g de fromage. Les valeurs sont, semble-t-il, sans relation étroite avec le $\mathrm{pH}$ de la pâte et les niveaux les plus élevés sont observés sur les échantillons 6 et 7 qui présentent la plus forte densité de mycélium.

\section{Mycélium}

Sur le mycélium, il a été possible de déterminer, pour la plupart des échantillons, l'activité sur les deux substrats aux pH 4,5-5,5 et 8,0. Les résultats sont regroupés dans la figure 1.

La valeur maximale est obtenue à $\mathrm{pH} 5,5$ sur tricaproïne, sauf pour l'échantillon 4 ; sur ce substrat, les niveaux relatifs d'activité à $\mathrm{pH} 4,5$, varient de 50 à $70 \%$ et à $\mathrm{pH} 8$ de 40 à 70 . L'activité sur tributyrine est trop faible pour être déterminée aux trois $\mathrm{pH}$ sur l'échantilon 5 et à pH 4,5 sur les échantillons 4 et 7 . Dans les autres cas, le rapport d'activité tributyrine/tricaproïne montre des variations importantes d'un échantillon à l'autre lorsque l'on compare les valeurs obtenues aux différents $\mathrm{pH}$ : pour les échantillons 1 et 6 , les rapports 


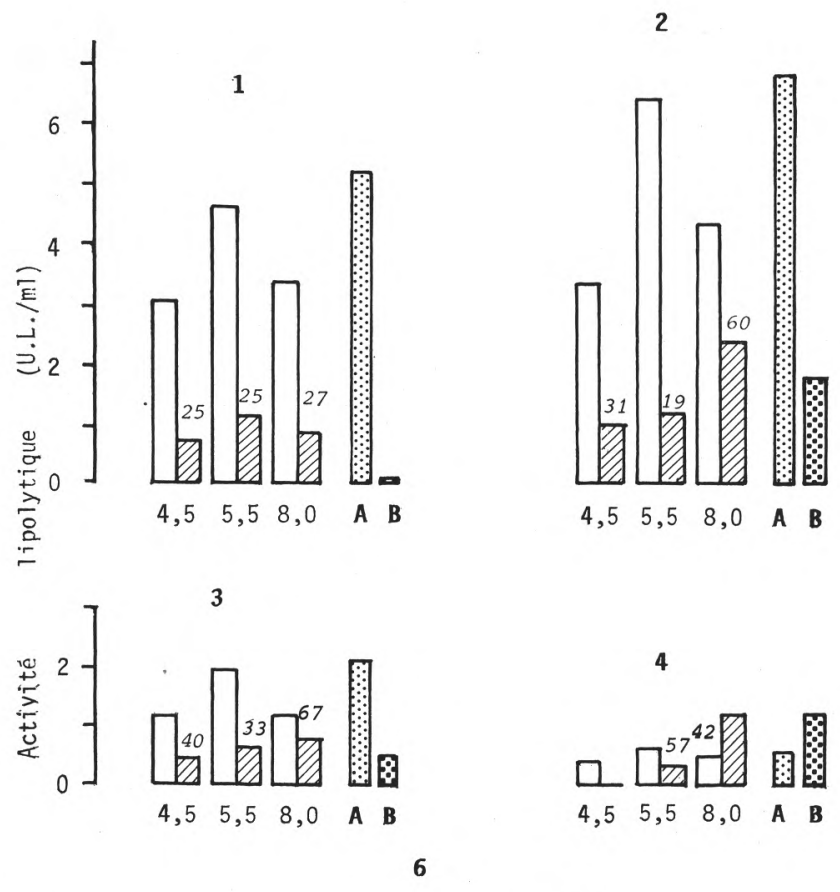


fig. 1

Activités lipolytiques de mycélium prélevés dans les fromages

à pâte persillée

Les activités ont été déterminées à $20^{\circ} \mathrm{C}$ au pH-stat.

En abcisses, les $\mathrm{pH}$ de détermination. Les nombres représentent, en \%, le rapport d'activité sur le substrat le moins favorable, pour chaque valeur de $\mathrm{pH}$. Substrat

$$
\bigotimes \text { Tributyrine } \square \text { Tricaproïne }
$$

Quantités calculées de lipases acide et alcaline (voir texte).

\section{A $Q$ Lipase acide ; B Lipase alcaline}

Echantillons (voir tableau 1).

1. Roquefort à 20 semaines d'affinage (souche 1 ).

2. Roquefort à 47 semaines d'affinage (souche 1 ).

3. Roquefort à 20 semaines d'affinage (souche 2 ).

4. Roquefort à 47 semaines d'affinage (souche 2 ).

5. Roquefort.

6. Saingorlon.

7. Bleu Bavarois.

fig. 1

Lipolytic activities of the mycelium gathered in the samples of blue veined cheeses

Activities determined at $20^{\circ} \mathrm{C}$ by $\mathrm{pH}$-stat method.

In abscisiae, $p H$ of determination. Numbers above diagrams give as $\%$ the activity obtained on the less favourable substrate, at each $\mathrm{pH}$ value.

Substrate

Tributyrins $\square$ Tricaproins

Calculated amounts of acid and alkaline lipase (Ref. text).

A $\because$ Acid lipase; $B$ Alkaline lipase

Samples (Ref. table 1).

1. Roquefort at the ripening time of 20 week (strain 1).

2. Roquefort at the ripening time of 47 weeks (strain 1).

3. Roquefort at the ripening time of 20 weeks (strain 2).

4. Roquefort at the ripening time of 47 weeks (strain 2).

5. Roquefort

6. Saingorlon

7. Bleu Bavarois

Commercial purchased samples. 
ne changent pas significativement avec le pH, de 20 à $27 \%$; pour les numéros 2, 3 et 7, l'activité sur tributyrine augmente lorsque le pH croît. Ainsi, à $\mathrm{pH} 8,0$, elle est supérieure à celle mesurée sur tricaproïne dans l'échantillon 7 et elle représente l'activité la plus forte dans le $n^{\circ} 4$.

\section{DISCUSSION}

Sur la base des résultats obtenus aux différents $\mathrm{pH}$, sur les deux substrats, on peut admettre que l'activité lipolytique du mycélium est la résultante de celle des deux lipases de Penicillium roqueforti. Les caractéristiques d'action de ces enzymes, déterminées sur des solutions partiellement purifiées, ont permis d'établir que les influences du $\mathrm{pH}$ et de la nature du substrat sur leurs activités respectives se traduisent par les coefficients mentionnés dans le tableau 2 (Lamberet et Menassa, 1983 ; Menassa et Lamberet, 1982). Il a été d'autre part vérifié que les rapports de spécificité vis-à-vis des deux substrats étaient indépendants du $\mathrm{pH}$.

Dans les échantillons 1 et 6 (fig. 1), la lipase acide semble seule présente car les activités relatives obtenues aux différents $\mathrm{pH}$ sur les deux substrats sont très voisines de celles de la lipase purifiée (Lamberet et Menassa, 1983). Il en est de même dans l'échantillon 5 où seule l'activité sur tricaproïne a pu être mesurée.

Dans les autres échantillons, le rapport activité sur tributyrine/ activité sur tricaproïne, au $\mathrm{pH} 8,0$ est supérieur à 0,3 . Ceci peut être considéré comme l'indice de la présence de lipase alcaline, plus active sur tributyrine que sur tricaproïne, présence en proportion d'autant plus importante que le rapport est plus élevé.

Une estimation des quantités respectives de l'une et l'autre enzymes peut être établie sur la base des mesures des activités à pH 5,5 sur tricaproïne $\left(\mathrm{N}_{1}\right)$ et à $\mathrm{pH} 8,0$ sur tributyrine $\left(\mathrm{N}_{2}\right)$ avec un système de deux équations à deux inconnues $\mathrm{A}$ et $\mathrm{B}$. A est la quantité de lipase acide exprimée par son activité sur tricaproïne au $\mathrm{pH}$ optimal 6,5, à $20^{\circ} \mathrm{C}$; B est la quantité de lipase alcaline exprimée par son activité sur tributyrine au pH optimal 9,5, à 20 $\mathrm{C}$. En considérant que $\mathrm{N}_{1}$ et $\mathrm{N}_{2}$ sont les sommes des activités de l'une et de l'autre enzymes, on peut écrire les deux relations qui prennent en compte l'influence du $\mathrm{pH}$ et celle de la nature du substrat :

(1)

$$
\begin{aligned}
& \mathrm{N}_{1}=\alpha \mathrm{A}+\mathrm{s} \beta \mathrm{B} \\
& \mathrm{N}_{2}=\alpha^{\prime} \mathrm{B}+\mathrm{s}^{\prime} \beta^{\prime} \mathrm{A}
\end{aligned}
$$

où les symboles représentent, pour la lipase acide :

$$
\alpha=\frac{\text { activité à } \mathrm{pH} \quad 5,5}{\text { activité à } \mathrm{pH} \quad 6,5}
$$


TABLEAU $2-T A B L E 2$

\begin{tabular}{|c|c|c|c|c|c|}
\hline & \multirow{2}{*}{ Substrat } & \multicolumn{4}{|c|}{$\mathrm{pH}$ de détermination } \\
\hline & & 5,5 & 6,5 & 8,0 & 9,5 \\
\hline Lipase acide & $\begin{array}{l}\text { Tricaproïne } \\
\text { Tributyrine }\end{array}$ & $\begin{array}{ll}0,9 & (\alpha) \\
(\alpha & \left.s^{\prime}\right)\end{array}$ & $\begin{array}{c}1 \\
0,215\left(s^{\prime}\right)\end{array}$ & $\begin{array}{c}0,72\left(\beta^{\prime}\right) \\
\left(\beta^{\prime} \mathrm{s}^{\prime}\right)\end{array}$ & \\
\hline Lipase alcaline & $\begin{array}{l}\text { Tricaproïne } \\
\text { Tributyrine }\end{array}$ & $\begin{array}{c}(\beta \mathrm{s}) \\
0,22(\beta)\end{array}$ & & $\begin{array}{c}\left(\alpha^{\prime} \mathrm{s}\right) \\
0,9\left(\alpha^{\prime}\right)\end{array}$ & $\begin{array}{c}0,40(s) \\
1\end{array}$ \\
\hline $\begin{array}{l}\text { (Les symboles correspondent } \\
\text { les équations (1) du texte). } \\
\text { Sources : }(5,6) \text {. }\end{array}$ & aux coefficients & és dans & \multicolumn{3}{|c|}{$\begin{array}{l}\text { (Symbols correspond to the coefficients used in the equations } \\
\text { (1) in the text). } \\
\text { From references } 5 \text { and } 6 .\end{array}$} \\
\hline
\end{tabular}


pour la lipase alcaline :

$$
\begin{aligned}
& \beta^{\prime}=\frac{\text { activité à } \mathrm{pH} 8,0}{\text { activité à } \mathrm{pH} 6,5} \\
& \mathrm{~s}^{\prime}=\frac{\text { activité sur tributyrine }}{\text { activité sur tricaproïne }} \\
& \alpha^{\prime}=\frac{\text { activité à } \mathrm{pH} 8,0}{\text { activité à } \mathrm{pH} 9,5} \\
& \beta=\frac{\text { activité à } \mathrm{pH} 5,5}{\text { activité à pH } 9,5} \\
& \mathrm{~s}=\frac{\text { activité sur tricaprö̈ne }}{\text { activité sur tributyrine }}
\end{aligned}
$$

En résolvant (1) on obtient le système d'équations :

$$
\begin{aligned}
& A=1,13 \mathrm{~N}_{1}-0,11 \mathrm{~N}_{2} \\
& B=1,13 \mathrm{~N}_{2}-0,19 \mathrm{~N}_{1}
\end{aligned}
$$

Les quantités respectives de lipases acide et alcaline calculées selon ces formules sur les suspensions mycéliennes analysées sont portées sur la figure 1 . On peut admettre que ces valeurs donnent une image représentative des teneurs en lipases des mycélium et donc des activités susceptibles de se manifester dans les fromages. En effet, l'activité à $\mathrm{pH} 8,0$ sur tributyrine n'a pu être détectée sur les fromages entiers que pour les échantillons 6 et 7 , le niveau d'activité traduisant l'abondance et la grande dispersion de la moisissure. Dans les autres échantillons, cette activité n'a pas été mise en évidence de manière significative à cause de la faible densité de mycélium (échantillons 1 et 2) ou de sa faible activité (échantillons 3, 4 et 5).

Une estimation précise et sûre des capacités d'action des deux enzymes exigerait de connaître l'influence des différents paramètres sur leur activité dans le milieu fromage, car les résultats obtenus " in vitro " ne sont pas nécessairement directement extrapolables. Cependant, on peut admettre, en première approximation, que la lipase acide qui apparaît comme la principale enzyme dans tous les échantillons, sauf dans le $\mathrm{n}^{\circ} 4$, doit avoir une action limitée dans le fromage, malgré un $\mathrm{pH}$ favorable, du fait de son faible niveau d'activité sur l'huile de beurre (3\% seulement par rapport à l'activité sur tricaproïne) (Lamberet et Menassa, 1983). En revanche, la lipase alcaline même présente en faible quantité et malgré des $\mathrm{pH}$ peu favorables pourrait avoir un rôle important dans certains échantillons car son activité sur l'huile de beurre est particulièrement grande : $60 \%$ par rapport à celle sur tributyrine (Menassa et Lamberet, 1982). 
L'influence de la souche de $P$. roqueforti peut être appréciée en comparant les résultats des échantillons 1 et 2 à ceux des $n^{\text {os }} 3$ et 4 . La souche 1 montre de forts niveaux d'activité de la lipase acide dans les deux échantillons et de la lipase alcaline seulement dans le fromage le plus affiné. La souche 2 révèle une activité alcaline plus précoce, avec une activité acide faible surtout dans l'échantillon le plus âgé.

En rapprochant les résultats relatifs aux quantités de lipases des observations organoleptiques, on constate qu'un goût piquant prononcé ou une légère saveur de savon est notée dans les fromages où la lipase alcaline est suceptible d'avoir le plus d'action, du fait d'un $\mathrm{pH}$ plus favorable ou d'une activité notable du mycélium : échantillons 3 - 4 et 7 . Dans l'échantillon 2, le goût paraît cependant équilibré malgré la plus forte activité alcaline de la moisissure. La très faible densité de mycélium dans la pâte ou la production tardive de la lipase alcaline pourrait sans doute l'expliquer.

\section{CONCLUSION}

La synthèse des deux enzymes apparaît nettement dépendante du facteur souche. La production des lipases est sans doute le reflet d'une aptitude à la lipolyse qui peut être plus ou moins grande mais, en même temps, elle est influencée par l'évolution du milieu, notamment celle du $\mathrm{pH}$, évolution largement dépendante des autres activités biochimiques. Les différences de niveaux relatifs d'action des deux lipases dans les fromages, sont très importantes ; elle doivent se traduire par de profils d'acides gras libres sensiblement différents (Imamura et Kataoka, 1963) et par suite par des caractères organoleptiques différents.

La méthode d'estimation proposée apporte un élément de réponse pour mieux comprendre le rôle de $P$. roqueforti dans le développement des qualités organoleptiques des fromages à pâte persillée et elle justifie l'intérêt que pourraient présenter des critères fondés sur les aptitudes biochimiques des souches en vue de leur sélection pour la fromagerie. Mais une connaissance plus approfondie des modes d'action des deux lipases dans les conditions d'affinage des fromages et, plus particulièrement, de l'expression de leur spécificité vis-à-vis des triglycérides du lait serait cependant nécessaire à une meilleure interprétation des résultats obtenus.

\section{Remerciements}

Nous remercions vivement $\mathrm{M}$. Assenat, Directeur $d u$ Laboratoire de la Société des Caves à Roquefort, pour la mise à notre disposition des échantillons de fromage de Roquefort. 


\section{Bibliographie}

Anderson (D. F.) and Day (E. A.) (1965). - Quantitative analysis of the major free fatty acids in Blue cheese. J. Dairy Sci., 48, 248-249.

Fujishima (T.), Ito (S.), Negishi (T.) and Fujino (Y.) (1971). - Studies on the lipids of cheese. II. On the composition of lipids and fatty acids in Blue cheese. Jap. J. Dairy Sci., 20, A5-A12.

HaLl (R.) et KosikowsKi (F. V.) (1970). - Composants et goûts caractéristiques des fromages à moisissure bleue faits avec du lait de vache et de brebis.

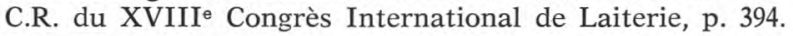

ImamuRa (T.) and Катаока (K.) (1963). - Biochemical studies on the manufacturing of Roquefort type cheese. II. Characteristics of lipases produced by Penicillium roqueforti. Jap. J. Zootech. Sci., 34, 349-353.

Lamberet (G.) and Menassa (A.) (1983). - Purification and properties of an acid lipase from Penicillium roqueforti (à paraître dans J. Dairy Res.).

Menassa (A.) et LAmberet (G.) (1982). - Contribution à l'étude du système lipolytique de Penicillium roqueforti. Caractères comparés de deux activités exocellulaires. Lait, 62, 32-43.

MoRRIs (H. A.) and JEZESKI (J. J.) (1953). - The action of microorganisms on fats. II. Some characteristics of the lipase system of Penicillium roqueforti. J. Dairy Sci., 36, 1285-1298.

Niki (T.), Yosнioкa (Y.) and Aмiкo (K.) (1966). - Proteolytic and lipolytic activities of Penicillium roqueforti isolated from Blue cheese. XVII Int. Dairy Cong., D, 531-537.

Vanbelle (M.), Vervack (W.), et Foulon (M.) (1978). - Composition en acides gras supérieurs de quelques types de fromages consommés en Belgique. Lait, 58, 246-260. 